\title{
ENFERMEDAD GANGLIONAR RESIDUAL TRAS QUIMIORRADIOTERAPIA CON INTENCIÓN RADICAL EN PACIENTES CON TUMORES DE FARINGOLARINGE
}

\section{Persistent nodal disease after chemo-radiotherapy with radical intention in patients with pharyngolaryngeal neoplasms}

\author{
Blanca P. GALINDO-TORRES; Mahfoud EL UALI-ABEIDA; María LLANO-ESPINOSA; Emilio \\ VIVES-RICOMÀ; Laura REBOLLEDO-BERNAD; Félix DE MIGUEL-GARCÍA; Rafael FERNÁNDEZ- \\ LIESA
}

Servicio de Otorrinolaringología. Hospital Universitario Miguel Servet. Zaragoza. España.

Correspondencia: togablan@gmail.com

Fecha de recepción: 21 de abril de 2017

Fecha de aceptación: 9 de junio de 2017

Fecha de publicación: 17 de junio de 2017

Fecha de publicación del fascículo: 1 de diciembre de 2017

Conflicto de intereses: Los autores declaran no tener conflictos de intereses

Imágenes: Los autores declaran haber obtenido las imágenes con el permiso de los pacientes

Política de derechos y autoarchivo: se permite el autoarchivo de la versión post-print (SHERPA/RoMEO)

Licencia CC BY-NC-ND. Licencia Creative Commons Atribución-NoComercial-SinDerivar 4.0 Internacional

Universidad de Salamanca. Su comercialización está sujeta al permiso del editor 
SUMMARY

KEYWORDS
Introduction and objective: Cervical nodal involvement is the main prognostic factor in squamous cell carcinoma of the head and neck. Salvage surgery is considered the most curative intervention for residual or recurrent disease after treatment with chemo-radiotherapy. The objectives of the study were to describe the epidemiological data of the patients, to evaluate the usefulness of the imaging tests, to analyze the survival, and to make a review of the current bibliography. Method: A retrospective study was carried out, including patients undergoing cervical lymph node surgery between January 2010 and December 2015, after radiological suspicion of persistent regional disease was found. All the patients had previously received chemo-radiotherapy treatment with radical intention due to a carcinoma of pharynx or larynx. Results: Thirty patients were included. The most common tumoral site was supraglottis (13). The most frequent histological result was poorly and moderately differentiated epidermoid carcinoma (10 cases in each). Sixty percent of the patients had stage IVa. The positive predictive value (PPV) of the CT scan was $55.26 \%$. Eighty-seven point five percent of the histologies confirming radiological diagnostic suspicion in a first surgery occurred in patients who had an early CT scan. Overall survival was $53.33 \%$; forty percent of the patients were free of disease at the end of the period. Conclusions: The low PPV of the CT scan makes its usefulness questionable as a method to assess nodal response to chemo-radiotherapy. It seems reasonable to propose selective lymph node surgery in selected patients.

squamous cell carcinoma; head and neck; chemo-radiotherapy; lymph nodes; salvage surgery

\section{INTRODUCCIÓN}

El carcinoma epidermoide de cabeza y cuello ocupa aproximadamente el $10 \%$ de los cánceres a nivel mundial, siendo diagnosticados más de medio millón de casos cada año [1].

La afectación ganglionar cervical metastásica constituye el principal factor pronóstico en estos pacientes; de ahí surge la necesidad de su control durante el tratamiento quimioradioterápico y el seguimiento posterior [2-5]. La cirugía de rescate se considera la intervención más curativa para la enfermedad residual 0 recurrente tras la quimiorradioterapia [6,7]; sin embargo, la selección de los pacientes candidatos es a menudo difícil. Además, las indicaciones y los beneficios en cuanto a la supervivencia son todavía anecdóticos por la limitada evidencia. Parece haber consenso en aquellos pacientes con enfermedad ganglionar inicial N1. Aquellos que tras el tratamiento con quimiorradioterapia presentan una respuesta completa, no requerirían cirugía cervical de rescate, mientras que en aquellos en los que hubiera una respuesta incompleta regional, sí que sería necesaria [8-10].

En cambio, la controversia aparece en pacientes con un estadio por encima de N1, ya que algunos autores han descrito una probabilidad de 3 a 7 veces mayor de enfermedad metastásica en pacientes N2 o mayor [11], así como un riesgo aumentado de recaída en pacientes N3 [12].

Evaluar la presencia de adenopatías residuales en pacientes tratados con quimiorradioterapia, tanto en la región patológica como en el tejido normal circundante, resulta en ocasiones extremadamente difícil por los cambios ocasionados por la inflamación. Para facilitar el diagnóstico, tenemos a nuestra disposición distintas pruebas de imagen como la tomografía axial computarizada (TAC), la resonancia nuclear magnética (RNM), la ultrasonografía (US), la tomografía por emisión de positrones con tomografía computarizada (PET-TAC) y la punción aspiración por aguja fina (PAAF). La prueba de imagen que utilizamos en nuestro servicio, por decisión en nuestro comité de tumores de cabeza y cuello es la TAC. Según Ojiri [13], los factores que podrían predecir el resultado patológico con esta técnica de imagen incluirían la baja densidad intranodal, la calcificación intranodal, la diseminación extracapsular y el tamaño de las adenopatías.

Los objetivos del estudio fueron: estudiar las características epidemiológicas de los pacientes a los que se les intervino de cirugía ganglionar cervical de rescate tras recibir tratamiento con quimiorradioterapia con intención curativa, analizar la utilidad de las pruebas de imagen realizadas, obtener los datos de supervivencia y realizar una revisión de la bibliografía actual.

\section{MATERIAL Y MÉTODO}

Se realizó un estudio observacional retrospectivo en el que se incluyeron los pacientes diagnosticados de carcinoma de faringe $o$ laringe que habían recibido tratamiento con quimiorradioterapia con 
intención radical, a los que se les intervino de cirugía ganglionar cervical de rescate en nuestro Servicio en el periodo transcurrido entre enero de 2010 al 31 de diciembre de 2015 por sospecha de adenopatía residual. La sospecha de la presencia de una adenopatía residual se objetivó en todos los casos mediante una TAC cervical. Se excluyeron los pacientes con metástasis cervicales de origen desconocido y pacientes que recibieron tratamiento quimio-radioterápico con intención radical con estadio inicial de NO y que posteriormente se les realizó cirugía ganglionar de rescate, por ser la adenopatía de nueva aparición. Se utilizó el programa estadístico SPSS para el análisis de los datos.

\section{RESULTADOS}

Se incluyeron un total de 30 pacientes, de los cuales un $93,33 \%$ (28) eran hombres y el $6,67 \%$ (2) restante mujeres. La media de edad en la fecha de la intervención fue de 61,60 años $(S D=8,373)$, con una edad mínima de 48 años y una máxima de 80 años. Se realizaron 38 intervenciones, ya que 8 de los pacientes precisaron 2 cirugías de rescate tras el tratamiento con quimiorradioterapia. De estos 8 pacientes reintervenidos, la segunda cirugía fue ipsilateral en 3 casos y contralateral en los otros 5.

La localización inicial predominante fue a nivel de supraglotis (13 casos), seguida de orofaringe (7), hipofaringe (5), nasofaringe (4) y glotis (1). El resultado histológico más frecuente fue de carcinoma epidermoide pobremente y moderadamente diferenciado (10 casos en cada uno), seguido del carcinoma indiferenciado (4), epidermoide bien diferenciado (1) y epidermoide no queratinizante (1). Hubo 4 casos en los que no se especificó el tipo de variante histológica.

Utilizando la clasificación TNM de la American Joint Committee on Cancer del año 2012 [14] como método de estadificación del tumor y sus metástasis, previamente al tratamiento, encontramos que el $56,6 \%$ (17) eran tumores avanzados (T3 o mayor), el $80 \%$ eran N2 y $10 \%$ N3, siendo el estadio más frecuente el IVa con el $60 \%$ (18) de los casos.

De los 30 pacientes que se intervinieron inicialmente por la sospecha de una persistencia de enfermedad ganglionar en la TAC tras finalizar su tratamiento con quimiorradioterapia, la anatomía patológica fue positiva sólo en 16 de ellos. De los 8 pacientes que precisaron una segunda cirugía, el resultado histológico fue positivo sólo en 5 de ellos (coincidiendo en todos ellos el resultado de positividad o negatividad con la primera cirugía). Es decir, de las 38 intervenciones realizadas, sólo se confirmó la sospecha de persistencia de enfermedad regional en 21, obteniendo así un valor predictivo positivo del $55,26 \%$.

Analizando el tiempo transcurrido desde el fin de la quimiorradioterapia hasta la aparición de una adenopatía sospechosa de malignidad en la TAC, observamos que la media fue de 438 días (14 meses), siendo este tiempo muy variable, desde 27 días hasta 5371 (14 años). Si dividimos el tiempo en 2 periodos, uno de menos de 12 semanas, y otro mayor de 12 semanas, encontramos que el $87,5 \%$ (14 de 16) de las histologías que confirmaron la sospecha diagnóstica en una primera cirugía ocurrieron en los pacientes a los que se les había realizado una TAC de forma precoz. Asimismo, de los 14 casos en los que la anatomía patológica descartó la presencia de enfermedad residual, el $57,14 \%$ (8 de 14) se dieron en estas primeras 12 semanas.

Únicamente se realizó una punción aspiración con aguja fina (PAAF) a uno de los 30 pacientes, tras la sospecha en la TAC, resultando positiva. La cirugía ganglionar cervical de rescate confirmó la presencia de 2 ganglios con enfermedad metastásica persistente.

Por otro lado, sólo se llevó a cabo un PET-TAC en 4 ocasiones, de las cuales, en 2 se confirmaba la sospecha de la TAC, y en los otros 2 no parecía patológico. La cirugía ganglionar cervical de rescate reveló un verdadero positivo, un falso positivo, un verdadero negativo y un falso negativo, si bien es cierto que el número reducido de exploraciones no permite extraer conclusiones sobre su utilidad diagnóstica.

La supervivencia global a los 5 años (Figura 1) fue del $53,33 \%$, ya que se produjeron 14 fallecimientos. La supervivencia específica fue del $60 \%$ puesto que dos de los pacientes fallecieron por motivos ajenos a la enfermedad. La supervivencia media fue de 43,33 meses. De los 16 pacientes que finalizaron vivos el periodo de observación, el $13,3 \%$ (4) tenían enfermedad, mientras que el $40 \%$ (12) permanecieron vivos y libres de enfermedad. 


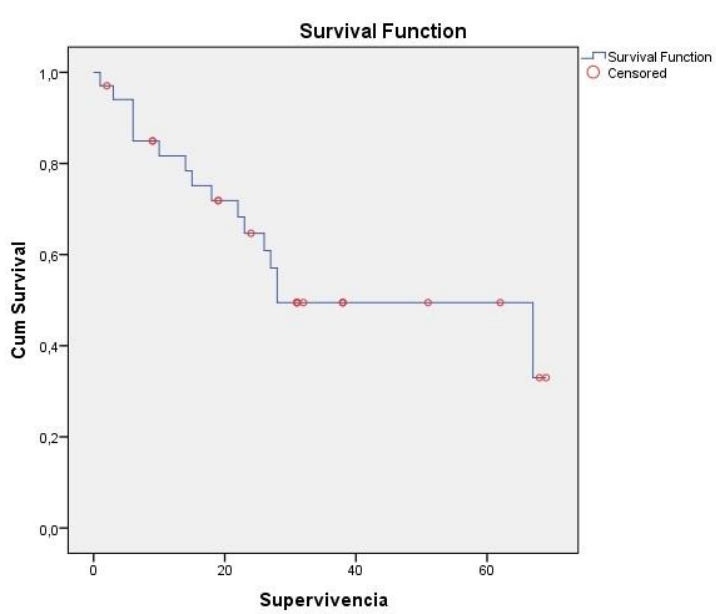

Figura 1. Supervivencia global a 5 años.

\section{DISCUSIÓN}

El éxito del tratamiento de la enfermedad ganglionar metastásica es un factor pronóstico para el control de los carcinomas epidermoides de cabeza y cuello en estadios avanzados [2]. A pesar de los progresos en su tratamiento, un número no desdeñable de pacientes presentan recidivas locorregionales o sistémicas en los 3 primeros años tras el tratamiento [3, 4]; cuando esto ocurre, la supervivencia global media es menor a un año [5].

El principal argumento para realizar una disección ganglionar cervical programada es mejorar el control regional [15], pero esto requiere un sistema de evaluación altamente eficaz que permita detectar las recurrencias o persistencias tumorales de forma precoz, con alta sensibilidad y especificidad, para evitar las complicaciones o secuelas postoperatorias que pudieran producirse en los pacientes que no precisaran la cirugía.

Los defensores de evitar la disección ganglionar cervical programada señalan que varias series han demostrado que la tasa de recurrencia ganglionar aislada es muy baja (entre un 0 y un 8\%) [8, 16-19], realizándola solo en los casos de una respuesta ganglionar incompleta tras el tratamiento con quimiorradioterapia. Además, la cirugía en pacientes con respuesta completa no se ha asociado con beneficios en la supervivencia [20,21]

Al revisar la bibliografía, no encontramos trabajos con los mismos criterios de inclusión que nuestro estudio, ya que otros autores realizaban el análisis en función del $\mathrm{N}$ o del estadio, y no de los pacientes intervenidos por sospecha radiológica de persistencia de enfermedad ganglionar como fue nuestro caso. En el caso de Nayak [11], se incluían los pacientes con enfermedad ganglionar N2 y sólo se intervenía al paciente en el caso de que hubiera una sospecha en el PET-TAC. En el caso de Karakaya [15], se incluían pacientes con tumores N3, y sólo se realizaba la cirugía de rescate en el caso de respuesta incompleta ganglionar según la TAC, la RNM o el PETTAC. Nishimura [2] incluyó a pacientes N2, y la cirugía sólo se realizaba a los pacientes con una PAAF positiva tras la aparición de una sospecha con US o PET-TAC. Por último, Kim [22] incluyó pacientes en estadios III y IVa, y decidía realizar la cirugía según distintos criterios radiológicos y resultado de una biopsia.

La aparición de este tipo de tumores en nuestro estudio aparece principalmente en varones, dato que coincide en todos los trabajos revisados [2,15, 22]. La localización del tumor más frecuente en nuestro caso fue en supraglotis $(43,4 \%)$, mientras que en otros trabajos predomina la localización en orofaringe o hipofaringe [2, 15, 22], o incluso base de lengua [11]. El resultado histológico predominante fue el de carcinoma escamoso moderada o pobremente diferenciado $(33,33 \%$ de cada uno), similar a los otros estudios [2, $15,22]$.

Analizando la utilidad de las distintas pruebas de imagen (TAC, RNM, US, PET-TAC y PAAF) descrita en la bibliografía revisada, encontramos que todas ellas tenían una especificidad por encima del $70 \%$ y un valor predictivo negativo por encima del $90 \%$, lo que permite confirmar con cierta seguridad los pacientes sanos [2, 11, 22]. El problema surge en que la sensibilidad y el valor predictivo positivo se ve muy reducido, pudiendo así incrementar el número de falsos positivos radiológicos y por tanto el número de cirugías innecesarias. La sensibilidad de la TAC/RMN, US, PET-TAC y PAAF varía entre un $44,4 \%$ [22] a $66,7 \%$ [2], 89,7\% [2], 51,7\% [2] - a $90 \%$ [22] y $68,2 \%$ [2] respectivamente según los estudios. El valor predictivo positivo de la TAC en nuestro estudio fue de $55,26 \%$, frente al $27,6 \%$ [2] a 36,4\% [22] de la TAC/RM, 33,3\% [2] de la US, 45\% [22] a 78.9\% [2] del PETTAC y $83,3 \%$ [2] de la PAAF de los otros trabajos. Este bajo valor predictivo positivo de 
las distintas pruebas de imagen puede hacer que pacientes sin enfermedad ganglionar residual se sometan a cirugías, en hasta el $45 \%$ de los pacientes en nuestro caso, enfrentándose a posibles complicaciones o secuelas postoperatorias sin mejorar su supervivencia. Por este motivo parece razonable la realización de vaciamientos ganglionares selectivos de las áreas con alta sospecha de persistencia de enfermedad residual.

En relación a la supervivencia, observamos que a pesar de que los pacientes incluidos en nuestro estudio tenían en principio peor pronóstico que los de los demás trabajos, por ser la sospecha de enfermedad ganglionar residual el criterio de inclusión presentaba unos datos similares a los de los otros autores (53,33\% frente al $51,4 \%$ a los 3 años descrita por Karakaya [15]). En este sentido, llama la atención que, aunque el SUV del PET-TAC no fue un criterio definitivo para intervenir a los pacientes del estudio de Kim [22], al finalizar el periodo de observación, detectaron una mejor supervivencia a los 3 años en pacientes que presentaban un SUV $<4,4(87,7 \%)$ respecto a aquellos con SUV $>4,4$ (56,9\%).

En cuanto a las utilidades de las distintas pruebas de imagen, Nayak [11] concluía que el PET-TAC tenía un alto valor predictivo negativo, lo que permitía seguir una actitud expectante si el resultado era negativo. Karakaya [15] por su parte, determinaba que la cirugía sólo debía realizarse en los casos de respuesta incompleta, ya que la tasa de recurrencia ganglionar aislada era muy baja en estos casos, y además estos pacientes no obtenían un beneficio en la supervivencia si eran intervenidos. Nishimura [2] describía una difícil valoración de la enfermedad locorregional tras el tratamiento con quimiorradioterapia mediante la TAC y la RNM, sugiriendo la posibilidad de realizar US 0 el PET-TAC pasadas 8 semanas tras el tratamiento, o la PAAF en el caso de discrepancia clínico-radiológica cuando se sospeche una respuesta incompleta. Por último, Kim [22] afirmaba que el PET-TAC obtenía unos mejores resultados para la detección de la recurrencia, realizándose pasadas de 10 a 12 semanas tras el fin del tratamiento, y que la cirugía permitía un dudoso control de la enfermedad a largo plazo con altas tasas de morbilidad.

\section{CONCLUSIONES}

El presente estudio confirma los excelentes resultados oncológicos a largo plazo, que el vaciamiento ganglionar cervical aporta en pacientes con enfermedad regional residual o recurrente tras un tratamiento con quimiorradioterapia. En nuestro trabajo, la supervivencia fue similar a la de otros estudios $(53,33 \%)$. Sin embargo, el bajo VPP $(55,26 \%)$ $y$ el alto porcentaje de falsos positivos que proporciona la TAC nos hace replantear su utilidad como método para valorar la respuesta ganglionar a la quimiorradioterapia. Por ello, para una mejor selección de pacientes, hemos propuesto incluir en nuestro protocolo de valoración de respuesta locorregional tras quimiorradioterapia otras alternativas, aisladas o combinadas, para valorar la indicación de la cirugía ganglionar de rescate, como serían el PET-TAC, la US y/o la PAAF. Finalmente, con el objetivo de reducir al mínimo las posibles complicaciones que derivan de la disección cervical, parece razonable plantear la realización de vaciamientos ganglionares selectivos en estos pacientes.

\section{BIBLIOGRAFÍA}

1. Ferlay J, Parkin DM, Steliarova-Foucher E. Estimates of cancer incidence and mortality in Europe in 2008. Eur $\mathrm{J}$ Cancer. 2010;46(4):765-81.

2. Nishimura G, Yabuki K, Hata M, Komatsu M, Taguchi $\mathrm{T}$, Takahasi $\mathrm{M}$, et al. Imaging strategy for response evaluation to chemoradiotherapy of the nodal disease in patients with head and neck squamous cell carcinoma. Int J Clin Oncol 2005; 21(4): 658667.

3. Lee JC, Kim JS, Lee JH, Nam SY, Choi SH, Lee SW, et al. F-18 FDG-PET as a routine surveillance tool for the detection of recurrent head and neck squamous cell carcinoma. Oral Oncol. 2007;43(7):686-92.

4. Ryan WR, Fee Jr WE, Le QT, Pinto HA. Positron-emission tomography for surveillance of head and neck cancer. Laryngoscope. 2005;115(4):645-50. doi:10.1097/01.mlg.0000161345.23128.d4.

5. Vermorken JB, Mesia R, Rivera F, Remenar E, Kawecki A, Rottey S, et al. Platinum-based chemotherapy plus cetuximab in head and neck cancer. New Engl J Medicine. 2008;359(11):1116-27. 
6. Hermann RM, Christiansen H, Rödel RM. Lymph node positive head and neck carcinoma after curative radiochemotherapy: a long lasting debate on elective posttherapeutic neck dissections comes to conclusion. Cancer Radiother 2013; 17:323331.

7. Mandapathil $M$, Roessler $M$, Werner JA, Silver CE, Rinaldo A, Ferlito A. Salvage surgery for head and neck squamous cell carcinoma. Eur Arch Oto Rhino Laryngol. 2014;271(7):1845-50.

8. Ferlito A, Corry J, Silver CE, Shaha AR, Thomas Robbins K, Rinaldo A. Planned neck dissection for patients with compLete response to chemoradiotherapy: a concept approaching obsolescence. Head Neck. 2010;32(2):253-61.

9. Brizel DM, Prosnitz RG, Hunter S, Fisher SR, Clough RL, Downey MA, et al. Necessity for adjuvant neck dissection in setting of concurrent chemoradiation for advanced head-and-neck cancer. Int J Radiat Oncol Biol Phys 2004;58(5):1418-23.

10. Robbins KT, Doweck I, Samant S, Vieira F. Effectiveness of superselective and selective neck dissection for advanced nodal metastases after chemoradiation. Arch Otolaryngol Head Neck Surg 2005;131(11):965-9.

11. Nayak JV, Walvekar RR, Andrade RS, Daamen N, Lai SY, Argiris A, et al. Deferring planned neck dissection following chemoradiation for stage IV Head and Neck Cancer: The Utility of PET-TC. Laryngoscope 2007 Dec;117(12): 2129-34.

12. Thariat J, Ang KK, Allen PK, Ahamad A, Williams MD, Myers JN, et al. Prediction of neck dissection requirement after definitive radiotherapy for head-and-neck squamous cell carcinoma. Int J Radiat Oncol Biol Phys 2012;82(3):e367-74.

13. Ojiri, Mancuso AA, Mendenhall WM, Stringer SP. Lymph nodes of patients with regional metastases from head and neck squamous cell carcinoma as a predictor of pathologic outcome: size changes at CT before and after radiation therapy. AJNR Am J Neuroradiol. 2002 Nov-Dec;23(10):1627-31.

14. American Joint Committee on Cancer. AJCC cancer staging manual, 7th ed. New York: Springer, 2012.
15. Karakaya E, Yetmen O, Colpan Oksuz D, Dyker KE, Coyle C, Sen M, et al. Outcomes following chemoradiotherapy for N3 head and neck squamous cell carcinoma without a planned neck dissection. Oral Oncol 2013 Jan;49(1):55-9.

16. Hamoir M, Ferlito A, Schmitz S, Hanin FX, Thariat J, Weynand B, et al. The role of neck dissection in the setting of chemoradiation therapy for head and neck squamous cell carcinoma with advanced neck disease. Oral Oncol 2012;48(3):203-10.

17. Forest $\mathrm{VI}$, Nguyen-Tan PF, Tabet JC, Olivier MJ, Larochelle D, Fortin B, et al. Role of neck dissection following concurrent chemoradiation for advanced head and neck carcinoma. Head Neck 2006;28(12):1099105.

18. Corry J, Peters L, Fisher R, Macann A, Jackson M, McClure B, et al. N2-N3 neck nodal control without planned neck dissection for clinical/radiologic complete respondersresults of Trans Tasman Radiation Oncology Group Study 98.02. Head Neck 2008;30(6):737-42.

19. Lambrecht M, Dirix P, Van den Bogaert W, Nuyts S. Incidence of isolatedregional recurrence after definitive (chemo)radiotherapy for head and necksquamous cell carcinoma. Radiother Oncol 2009;93(3):498-502.

20. Cannady SB, Lee WT, Scharpf J, Lorenz RR, Wood BG, Strome M, et al. Extent of neck dissection required after concurrent chemoradiation for stage IV head andneck squamous cell carcinoma. Head Neck 2010;32(3):348-56.

21. Goguen LA, Posner MR, Tishler RB, Wirth LJ, Norris CM, Annino DJ, et al.Examining the need for neck dissection in the era of chemoradiation therapy for advanced head and neck cancer. Arch Otolaryngol Head Neck Surg 2006;132(5):526-31.

22. Kim R, Ock CY, Keam B, Kim TM, Kim JH, Paeng JC, et al. Predictive and prognostic value of PET/CT imaging postchemoradiotherapy and clinical decisionmaking consequences in locally advanced head and neck squamous cell carcinoma: a retrospective study. BMC Cancer 2016 Feb 17; $16: 116$. 